\title{
Synergies and trade-offs between OHS and productivity: an assessment method to support the design of new interventions
}

\author{
A. Rosti, P. Trucco, D. Masi \\ Politecnico di Milano, Milano, Italy
}

\begin{abstract}
Although several studies asserted the economic value of OHS (Occupational Health \& Safety) interventions, the expected return is hardly comparable with productivity related interventions. Therefore, Small and Medium sized enterprises (SMEs) tend to favour productivity related interventions, trying to achieve a trade-off between OHS and productivity. The present study proposes a method for a better understanding of the multifaceted relationship between OHS and productivity, with the purpose of getting a more accurate estimation of potential trade-offs and synergies between the two dimensions, and of supporting management in the design of interventions. The method uses a selected set of leading and lagging performance indicators and a semi-quantitative assessment of the expected effects. The method has been refined and validated through case studies, and the results of the empirical work highlight the ability of the method of adding value to current practices, its high adaptability and scalability, and a reasonable implementation cost, and suggests the advantages further exploring a deeper integration between OHS and productivity.
\end{abstract}

\section{INTRODUCTION}

The urgency of intervening on Occupational Health \& Safety (OHS) is widely acknowledged: the Italian National Institute for Insurance against Accidents at Work, for instance, reported an increasing total cost of accidents, despite a reduction of the number of injuries at national level (Inail-Contarp, 2012).

The debate on the economic value of Investments in Occupational Health \& Safety (OHS) produced contrasting results. Some studies highlight the possibility of Investments in OHS for reducing the accidents rate in a cost effective way (see e.g. ISSA, 2011) (Tompa et al., 2009). Other studies comparing OSH and productivity related interventions questioned the possibility of gaining direct benefits (Hasle, Bojesen, Jensen, \& Bramming, 2012) and highlighted how the Return on Investment of OHS interventions is lower, more difficult to estimate, and uncertain, also exposed to a high risk of failure (Cagno, Micheli, Masi, \& Jacinto, 2013; Cohen, A., Colligan, 1998; De Greef \& Van den Broek, 2004).

\section{STUDY OBJECTIVES AND METHODOLOGY}

The present study proposes a method for a better understanding of the multifaceted relationship between OHS and productivity, with the purpose of getting a more accurate estimation of potential tradeoffs and synergies between the two dimensions, and of supporting management in the design of interventions.

The method has been developed through a critical combination of selected studies on the relationship between OHS and productivity, and refined and validated through a set of case studies in different European companies.

The paper is structured as follows: in section 3, an overview of the literature on the relationships between OHS and productivity is presented. In section 4 , the method for a better understanding of the relationship between OHS and productivity is introduced; in section 5, the results of the empirical work are summarised and finally in section 6 some conclusions are taken.

\section{OHS AND PRODUCTIVITY: AN OVERVIEW OF THE LITERATURE}

In order to get a preliminary understanding of the relationship between OHS and productivity, a review of the literature has been undertaken.

The search employed keywords such as "OHS" and related synonyms combined with "Productivity" and related synonyms. The databases used were Scopus and Web of Science. Other papers were added by cross-referencing, and retrieved through Google Scholar. 
The papers were selected based on the relevance for the analysis relationship between OHS and productivity, with a focus on empirically validated studies that highlight the mechanisms generating synergies or trade-offs between OHS and productivity.

Using these criteria, a selected number of papers were analysed and presented in the following sections. These studies have been classified into studies presenting a negative impact of OHS on productivity, studies presenting economies of scope, and studies analysing learning economies.

\subsection{Negative impact}

To date only a few authors tried to categorize relationship between OHS and productivity. They generally adopted an "academic" perspective (Shearn, 2003) and highlighted how an empirically validated and deeper understanding of the mechanisms creating synergies and trade-offs is necessary. A first group of authors argue that OHS practices have negative implications for productivity outcomes, and productivity practices have negative implications for OHS outcomes. For instance, Zohar (2000) posits that there is a trade-off between organizational goals such as quality improvement and cost reductions and safety goals such as accident reduction. Hasle et al. (2012) reviewed the scientific literature on the effects of lean on the working environment and employee health and well-being, concluding that there is strong evidence for the negative impact of lean on both the working environment and employee health and well-being in cases of manual work with low complexity.

\subsection{Economies of scope}

A second group of authors argue that the combined effect of productivity and OHS practices can generate synergies or economies of scope. The practices generating synergies between OHS and productivity involve both the strategic level and the operational level.

At a strategic level, various aspects have been considered, such as the business strategy (De Greef et al., 2004), the culture of the enterprise (Veltri et al., 2013), the management system (Veltri et al., 2013), and the organizational configuration of the enterprise (Kirsten, 2010).

At an operational level, the synergies between OHS and productivity deal with the working environment, with the way of realizing the product and with the way of managing the work of the employees. A simple example is the case of safer workplaces that increase the overall OHS but also increase quality and productivity (Tompa et al., 2009). Another synergy between OHS and productivity exploits the possibility of optimizing the way in which a task is undertaken in light of the human capabilities (Dan, Falck, \& Ortengren, 2010).

Meaningful examples are the study of ILO (2010) that proposes a set of practical and easy to implement solutions for improving safety, health and working conditions while improving productivity. The study proposes a clear analysis of the mechanism of improvement of OHS and of the mechanisms of improvement of productivity related to the different interventions. More recently, Adaramola (2012) examines mental and physical pressures that workers bear at work. The author discusses how on the-job stress affects a person's capabilities and productivity, and how such pressures lend to higher incidences of accidents at work.

From different angles and perspectives, these studies agree on the conclusion that OHS measures can influence company performance positively and that contribute to the company goal, essentially through the creation of better working conditions, working climate, and improved organisational process.

The critical issue in capturing the benefits of this synergy is the correct definition of the scope of intervention. Indeed, a single practice can produce a wave of effects that is hard to evaluate ex-ante, since most of the times the actual scope is not fully clear at the beginning (Dance, Williams, Veltri, \& Tx, 1996). Moreover, different types of benefits may require different timeframes before showing the full impact, thus introducing an additional hurdle to the analysis. (Nieuwenhuis \& Lyons, 2002) suggest that the synergies based on economies of scope can provide only limed improvement.

\subsubsection{Learning Economies}

The third group of studies describe the way in which OHS practices improve productivity practices through learning economies. The share of relevant experiences and the mutual use of lessons learned can indeed make both OHS and productivity interventions more effective. We will refer to this kind of influence with the term "learning economies".

The learning economies are mainly described in the literature dealing with management systems such as ISO 14001 for environmental issues, ISO 9001 for quality and OHSAS 18001 for social issues (Boiral and Gendron, 2011). There are many interdependences between the different management systems and therefore lessons learned from quality management and the management of working conditions can be valuable for environmental management and vice versa. Zwetsloot (1995) identifies three types of synergies that have to do, respectively, with common aspects, similar organizational principles (both at the system and the element level) and the relationship with general management. 
The accounting system is the most common process influenced by learning economies.

Learning from past practices enable a more efficient allocation of resources. For example, in case of first introduction of new practices, managers have to estimate the effort and, later on, they may need to take additional measures, resulting in a loss of productivity and usually in a trade-off between economic and safety results. Instead, in case of a second implementation, the company is able to plan from the very beginning the additional measures, therefore the implementation would be more efficient, resulting in better economic performance as well as higher OHS outcomes.

In the end, a lesson learned enables to improve the design and estimation process of an intervention, resulting into a better understanding of the process and a more efficient allocation of resources.

\subsubsection{Synergies based on Integration}

Synergies occur also by integration of two or more processes. This practice results into a cost reduction, but also in an increase of performance (on both OHS and productivity).

The most common practice of integration between OHS and productivity concerns Management Systems. In fact, when the two are separated and independent, a substantial trade-off arises, since every system tries to achieve its own performance targets, disregarding the potential detrimental effects on other performance dimensions.

This practice requires some preconditions:

- The presence of a structured Safety Management System.

- A systematic collection of all the OHS's data, not only the most explicit ones.

- The responsibility of both Productivity and Safety and Health have to be committed to a single chain of command, reducing the chances of internal compromise.

For these reasons, the integration of management systems usually is profitable only for Medium-Large Enterprises, since they have enough resources for successfully implementing integrated systems and exploiting the benefits.

\subsubsection{Synergies based on Marketing Value}

The adoption of OHS practices can also increase the number of potential customers. Indeed, nowadays many international companies require to suppliers the acquisition of OHS certifications based on international standards.

This type of synergy is not directly related to productivity, but it links OHS to the profitability of the company; is the market justification of OHS interventions that paves the way to all the other forms of synergy.
In the end, this effect is the most contingent synergy, since the benefits are influenced not only by the internal factors, but also by the external conditions of the market.

\section{MODEL FOR A JOINT EVALUATION OF OHS AND PRODUCTIVTY OF INTERVENTIONS}

\subsection{Scope and objectives of the model}

The model aims to provide a new method for the management and evaluation of the synergies between OHS and Productivity in a new intervention.

The analysis of the different types of relationships (synergies and trade-offs) between productivity and OHS suggests that only an integrated and multidisciplinary analysis is effective in order to estimate accurately the outcome.

The model is based on KPIs, since these are very common among managers and are easy to adapt to the company needs. Moreover, KPIs are often used for both productivity and OHS evaluation, resulting into a common feature able to link the two dimensions.

This model is meant to be considered as part of the design process, since it yields more benefits when implemented together with the traditional design processes already present in the company. In this way, the tool provide useful information and input for the refinement of the intervention, in order to achieve a better exploitation of the interdependences between OHS and productivity.

However, the outcome of this method depends on how the intervention is undertaken, since the company condition influence the results.

Therefore the framework should be embedded in the iterative process of the intervention evaluation, in order to achieve satisfying performances.

\subsection{Structure of the framework}

This framework is based on a stepwise procedure (Figure 1) able to guide a cross functional team in the analysis and management of the synergies between Productivity and OHS.

The procedure starts from the analysis of the unbundled effects and concludes with the integration of the results into the traditional project management.

The company has to undertake these steps for every iteration of the intervention design process, in order to yield the maximum benefit. However, even the implementation of only the first steps should provide a valuable improvement for managing the interdependences. 


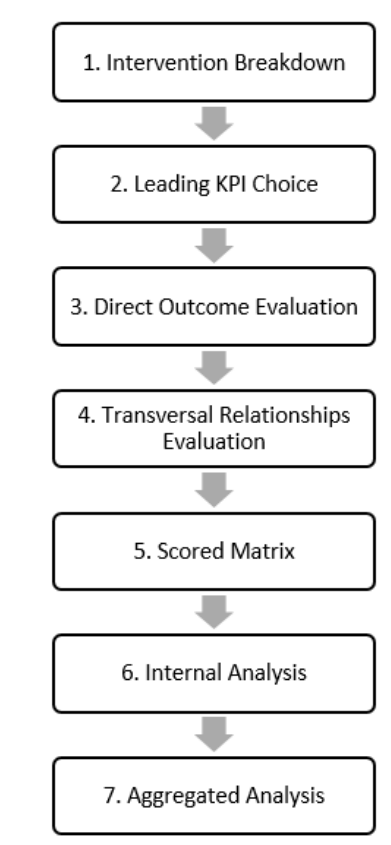

Figure 1: Stepwise procedure

\subsection{Steps of the method}

\subsubsection{Intervention Break Down}

Usually an intervention includes the introduction or changes of several practices. Therefore, the first step is to unbundle the whole intervention into elementary and easy to analyse practices/changes.

We can define in this research an elementary change as a part of intervention that cannot be further divided without jeopardizing the benefits.

This step allows identifying which are the main elements of change, whether productivity or safety related.

An elementary change can affect any aspect of the organization: from the process tasks to the hierarchical structure, from the technology to the human resources aspects. For this reason, the project manager has to involve managers from all the functions affected in order to evaluate the intervention.

\subsubsection{Leading KPIs Choice}

After the unbundling of the intervention, it is possible to decide how to implement the new practices. Indeed each elementary practice/change can be translated into variations of leading performance indicators.

A Leading Performance Indicator (Lead) is an indicator that measures the effort, the choices or the input of the strategy. Usually they are used as a proxy to the future expected results (Podgórski, 2015).

In practice, the next step is to choose which Leading Performance Indicators (Lead) would undergo a change. At this moment is not relevant whether the KPI is OHS related or not.
This method facilitates both the analysis as well as the implementation of the intervention, since it offers a new framework to identify the decision variables actually involved but it is also useful to integrate and align the decisions over the same variables.

Indeed different elementary changes can involve the same Leading KPI. Moreover, different elementary practices might require opposite interventions on the same Leading Performance Indicator, resulting in an incoherent implementation.

Therefore, we can see that, as a by-product of this protocol, managers can graphically check whether the intervention is well-designed or not.

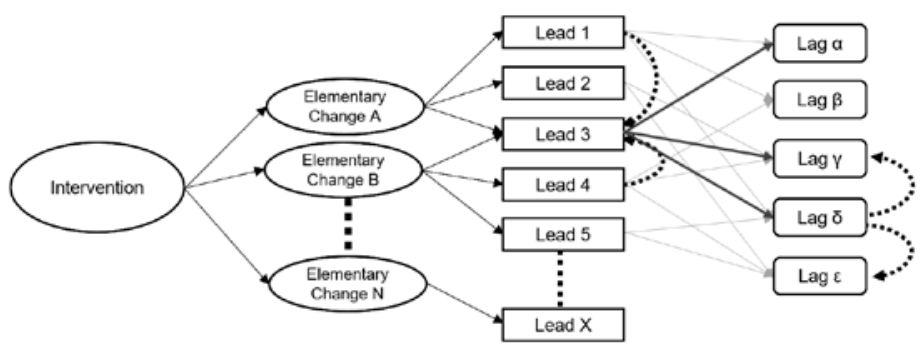

Figure 2: KPIs relationships

\subsubsection{Direct Outcomes evaluation}

The next step is to link the decisional variables (Lead KPIs) to the expected outcome. In practice, managers have to check for each Leading KPI which Lagging indicators are affected.

Lagging KPIs can guide managers to the identification of the variables influenced.

A lagging performance indicator (Lag) is a KPI that measures the outcome of a practice. Indeed it measures the results of something already happened (Podgórski, 2015). These indicators are widely used for the evaluation of the OHS outcomes, since they represent the actual improvements caused by the new practices; but in this case these indicators are used for the analysis of transversal relationships.

This step allows the management to identify and integrate the outcome of every dimension. This analysis identifies the cause-and-effect relationship and graphically highlights the variables influenced directly.

Because of the nature of these KPIs, the most important Lagging indicators will be affected by the many of the Leading indicators, since usually the practices implemented in a single intervention have a similar scope and aim, however this step aims to identify also the less evident relationships between indicators.

\subsubsection{Transversal Relationships Evaluation}

After the identification of the direct relationships, it is necessary to find out which transversal relationship, if any, is present. Indeed a Lead KPI may influence another Lead KPI resulting in an indirect ef- 
fect on the final lagging indicator, as well as the outcome of a Lagging Indicator may influence the results of another Lagging indicator (Figure 2).

This traversal link occurs when two indicators of the same kind affect each other. In other words, whenever the way a practice is implemented influences the way another variable (Leading) is implemented or whenever the outcome of a practice affects the outcome of other practices.

Moreover, the traversal relationship between Leading Indicators can affect indirectly the final Lagging indicators.

In order to carry out this step, managers have to identify which practices have the same scope or area of influence. While, to identify the dependencies between outcomes, managers have to verify the presence of a cause-effect relationship between each couple of Lagging KPIs.

\subsubsection{Scored Matrix}

In case of complex interventions, the number of indicators will increase exponentially, resulting into an enormous number of relationships to analyse. For this reason, it is useful to evaluate which Leading Performance Indicators have greater impact on the outcome.

The selections is based on two dimensions: "level of influence" and "level of expected effect".

The level of influence refers to the number and type of interdependences originated by the KPI. The level of expected effect, instead, is more complex to calculate. It refers to how much it affects the other KPI. In this way, we can plot every Leading Performance Indicator into a Matrix, in order to score and select the most relevant relationships based on wideness of the scope and magnitude of the effects.

Indicators in the top-right corner are the most important since they affect many factors with relevant magnitude, and we will call them as "very effective KPIs". Therefore, they require further studies in order to exploit the possible synergies as well as manage the trade-off.

\subsubsection{Internal Analysis}

After the selection of the KPIs to be analysed, we can proceed to the actual analysis and classification of the links between KPIs, accounting both direct and indirect effects.

For every "very effective KPI" we need to analyse how it affects both Productivity and OHS metrics. Because of the mixed nature of this step, it is advisable that a cross-functional team, coordinated by a project manager, may lead this analysis.

In practice, the managers have to follow the method in order to support a more accurate estimation of the effects.

When a Leading KPI selected through the matrix affects others KPIs only positively, the framework indicates a possible synergy for the company. In- deed, managers may exploit this positive relationship between Productivity and OHS promoting the change in that KPI or introducing now practices based on the very same Leading KPI, especially when transversal relationships are present.

In the same way, when the framework highlights a Leading KPI with only negative implications, managers have to prevent any increase in that indicator. In this situation, this tool is able to isolate the negative component from the whole intervention, allowing manager to prevent or at least reduce changes in that KPI. Therefore, even in case of negative relationships, this method provides managers a guide towards a better performance.

However, many Leading KPIs' changes involve both positive and negative effects. In this situation, the main issue is to account correctly the overall outcome.

When these conflicting outcomes are in the same dimension (either OHS or Productivity), then managers can estimate the overall result as the sum of the single outcomes. The same procedure cannot be undertaken when the conflicting effects are in different dimensions (for instance positive on Productivity and negative on OHS) since the effects are completely different and usually are measured in different ways (Productivity outcome is more defined and qualitative, while safety is based on qualitative estimations of risks). In this case, the outcome will have a double dimension.

Still, having the list of all the relationship between Leading KPIs and Lagging KPIs is a powerful tool. Indeed the same Lagging KPI can be affected in contrasting ways, in order to achieve a positive outcome.

At the end of this step, the managers identified and evaluated all the synergic relationships, regardless being interdisciplinary or not.

Therefore, the results can be integrated into a table that states for every link the effect and the type of relationship.

\subsubsection{Aggregated Analysis}

The previous steps unbundled the intervention and analysed the relationships and the dependences between KPIs, the next step is to integrate these results into the traditional evaluation process in order to achieve a more accurate estimation of the outcomes.

This process indeed aims to extend the intervention evaluation process currently adopted by the company, trying to comprehend the interdisciplinary relations and integrating the results.

This approach allows therefore a wider implementation of this tool, since it does not require radical changes in the internal processes.

The overall analysis process, therefore, starts from the traditional (and independent) evaluation of the variations (before/after analysis) of both OHS 
and Productivity outcomes; in the meanwhile, managers have to use this tool, in order to improve the design of the intervention as well as better estimate the overall outcome.

After both the analysis are completed, managers have to integrate the outcomes with this aggregated analysis step.

Indeed, the results of the traditional/current design process must be adjusted according to the analysis of the relationship developed in the previous steps. In practice, managers have to use the semiquantitative effects-table to fix and adjust the outcome of the traditional evaluations, linking the OHS performance with the Productivity ones.

\section{TEST AND EVALUATION OF THE FRAMEWORK}

The research required a critical review of the results in order to demonstrate that the new framework can be applied to real situations.

For this reason a series of test cases were conducted, to verify the scientific accuracy of the framework. Afterwards, the validation proceeded through the gathering of a company feedback on the research, in order to evaluate the actual added value in a real-life implementation.

\subsection{Four test applications in different industrial contexts}

In order to test the applicability of the framework and deliver to the companies a solid and refined version of the framework, four test cases were carried out.

This step aims to evaluate the theoretical functionality of the framework based on past interventions, before gathering the company feedback.

Since some of the test cases regard past or already completed interventions, it was not always feasible to fully implement the framework; however the method was adapted in order to make it applicable also in cases of limited or incomplete information, that is a rather common practical condition.

The cases were conducted in four different industries. The first application was inside an Italian supplier of printing machines. The second case was within a plant of a multinational specialised in presses and compressors.

The third application was held in plant owned by a swiss company specialized in textile robotics.

Finally, the last application was held in an electrical pane manufactures.

This case of study provided support to the scientific usefulness of the proposed framework; indeed the method enables a more accurate estimation of direct and indirect effects alike, resulting into an improved intervention-design process.
Table 1: Test Cases

\begin{tabular}{|c|c|c|}
\hline Test Case & Products and Industry & Type of Intervention \\
\hline Elettra s.r.l. & $\begin{array}{c}\text { Automatic machinery for } \\
\text { Printing }\end{array}$ & Safety policies redesign \\
\hline Stäubli Italia S.p.A & Textile Robotics & $\begin{array}{l}\text { Assembly process } \\
\text { redesign }\end{array}$ \\
\hline Ingersoll-Rand & $\begin{array}{c}\text { Presses and compressors for } \\
\text { Drilling }\end{array}$ & Cost reduction of tests \\
\hline Electrical Manufacturer & $\begin{array}{l}\text { Electrical low voltage } \\
\text { Components }\end{array}$ & Layout Redesign \\
\hline
\end{tabular}

Despite the positive results of the applications, the test cases highlighted new critical points and opportunities for improvement, involving changes and enhancement to the framework.

For instance during the analysis of the compressor manufacturer case two critical problems occurred. When the project scope is restricted, the tool yields limited benefits; indeed, its effectiveness is strictly related to the number of relationships among KPIs. Therefore, the author modified the framework in order to extend its adaptability. Another issue observed is the definition of the links between elementary changes and Lagging KPIs. Indeed, some interventions seems to yield no effect on any KPI, for this reason the direct-relationship analysis step has been redeveloped, in order to be more structured and easy to implement.

Finally, one more issues occurred during the electrical panel manufacturer case: whenever the factors are qualitative, it is complex to identify the adequate KPI. For this reason, the author added new indicators that can provide a support for the qualitative estimation.

Table 2: Case results

\begin{tabular}{|c|c|c|}
\hline \multicolumn{1}{|c}{ Test Case } & \multicolumn{1}{c|}{ Critical Issues } & Improvements made \\
\hline $\begin{array}{c}\text { Ingersoll-Rand and } \\
\text { Stäubli }\end{array}$ & $\begin{array}{c}\text { Limited benefits when the } \\
\text { project's scope is restricted }\end{array}$ & $\begin{array}{c}\text { Streamlining the analysis } \\
\text { process }\end{array}$ \\
\hline $\begin{array}{c}\text { Electrical panel } \\
\text { manufacturer }\end{array}$ & $\begin{array}{c}\text { Hard implementation when the } \\
\text { factors are qualitative }\end{array}$ & Introduction of new KPIs \\
\hline Ingersoll-Rand & $\begin{array}{c}\text { The link between elementary } \\
\text { interventions and Lagging KPIs } \\
\text { is not always obvious }\end{array}$ & $\begin{array}{c}\text { Redefinition of the direct } \\
\text { relationships analysis }\end{array}$ \\
\hline
\end{tabular}

\subsection{Critical evaluation by practitioners: protocol and results}

In order to evaluate the framework, the author designed a specific protocol of validation. The protocol was in the form of a questionnaire, made up of several parts and administered to leading companies, in order to evaluate the perception of the problem on the field, to evaluate the comprehensiveness of the factors presented in the framework and to evaluate the outcome of the tool in terms of usability and added value. In the end, the final step requires an explanatory case in order to evaluate and compare the results with the ex-ante analysis. 
The protocol was given to a multinational company, leader in the automotive parts industry.

Both OSH manager and Industrial Engineering Team manager studied the framework and the method, providing a critical analysis.

The management stated that the method is "quite enough usefulness and feasible within the company"; moreover they declared that all the data required for the implementation is already available (except the data for economic evaluation, since they prefer to implement that step in other ways).

However, they also highlighted that, if the new proposal is implemented, an increase in effort is required, but the benefits should be worth the increase at least for medium-to-large decisions.

In addition, they stated that this framework is more comprehensive than their actual evaluation process, and they might use it, if it were simplified (removing some steps like the matrix and the economic evaluation) and if it were fine-tuned for companies with a project-based production like Delphi.

Indeed the proposed framework has some redundancies in case of mixed production lines and does not considers some peculiarities of project-based production (e.g. some clients may require specific production methods, introducing further limitation to the decision process).

Regarding the company mixed production process, the managers proposed a new approach for the implementation of the framework. In particular, they highlighted that the analysis could start with the evaluation at local level (considering only one product) and only afterwards aggregating the results, in this way an implementation within complex multiproducts lines should be feasible and, theoretically, it should grant satisfying performance for the estimation and management of the synergies between productivity and OHS.

Finally, the managers perceive this method as an extension of their current decision process, since it could provide additional contribution for a better estimation of the results. Moreover, the interviewed managers highlighted the possibility of implementation for this method, after some adaptations such as the introduction of different dimensions (e.g. Quality-Productivity, TacktTime-OHS, etc.), proving that the framework is enough structured to be generalised into an ever more comprehensive tool. The interviewed appointed the "scored matrix" as a low value-adding step, especially considering the effort required to gather the opinions from the different management functions.

In the end, they perceived the analysis of transversal interactions as very useful and important, since it can disclose "hidden" interactions.

Moreover, the interviewed managers proposed to apply the tool not only to evaluate interventions, but also to choose the right alternative within a practical decision. For instance, when planning a new produc- tion line, frequently they have to make options between fully-automated vs semi-automated machines, and this tool appears to be useful for this purpose. At this stage, identifying and analysing potential interactions might be important and useful for the decision process.

\section{CONCLUSIONS}

In conclusion, the research highlighted the relationships between productivity and OHS, proving the importance of evaluating productivity gains when designing OHS interventions. The relationships between productivity and OHS is evaluated through an analysis of the synergies and trade-offs between the two dimensions.

The resulting method provides allows a better estimation of the safety implications in new interventions. In particular, the research demonstrated that OHS and productivity are two dependent variables, but the most common frameworks for the estimation of OHS outcome are not able to identify effectively the interdependences between the two dimensions, and therefore companies fail to achieve the expected outcomes.

The proposed method categorizes synergies and trade-offs, thus allowing a more comprehensive and accurate estimation of the integrated outcome.

\subsection{Contributions and limitations}

The main original contribution of this the tool is the use of Leading LPI and Lagging KPI, used in systematically linking the KPIs to synergic effects.

Moreover, the aggregation of the productivity and OHS outcomes within the company interventiondesign process allows the achievement of an integrated outcome, rather than two different outputs (OHS and Productivity).

The case studies and the company feedback highlighted few limitations. First of all, the framework is based on an extensive use of performance indicators, however not all the innovations and procedure intervention can be effectively measured with KPI, resulting into an inability to deploy the tool within strategic or qualitative analysis. Moreover, due to the lack of the temporal dimension, the tool is not suited to estimate the magnitude and effects of longterm learning economies.

\subsection{Future developments}

At the moment, despite the positive results of the framework, it is possible to highlight features and areas of the method that can be further developed, in order to achieve an even more comprehensive analysis. One possible trend of future development could be the introduction of a third dimension. In particu- 
lar, the addition of a quality-based dimension or a temporal dimension could better resemble the actual span of decision of the company.

Furthermore, the framework would benefit from the validation of the related method in a larger number of company cases. In particular, the implementation of this framework into a large real-life project could further confirm the benefits of the method, and maybe facilitate the adoption rate in other companies.

Finally, the proposed framework could be adapted and prove its usefulness beyond its scope of application. Indeed, the framework could be adopted to compare the outcome of interventions in the context of lean manufacturing, compared to other different contexts or approaches to safety, as well as, managers may implement conversely the method in order to disclose the causes of an unexpected effect.

\section{REFERENCES}

Adaramola, S. S. (2012). Job stress and productivity increase. Work (Reading, Mass.), 41 Suppl 1(SUPPL.1), 2955-8. http://doi.org/10.3233/WOR-2012-0547-2955

Bohle, P., \& Quinlan, M. (2000). Managing Occupational Health and Safety: A Multidisciplinary Approach. Retrieved from http://books.google.com/books?hl=it\&lr=\&id=xNKqMjs 4c28C\&pgis $=1$

Boiral, O., \& Gendron, Y. (2011). Sustainable Development and Certification Practices : Lessons Learned and Prospects, 347(October 2010), 331-347.

Cagno, E., Micheli, G. J. L., Masi, D., \& Jacinto, C. (2013). Economic evaluation of OSH and its way to SMEs: A constructive review. Safety Science, 53, 134-152. http://doi.org/10.1016/j.ssci.2012.08.016

Cohen, A., Colligan, M. J. (1998). Assessing Occupational Safety and Health Training, A literature Review. National Institute for Occupational Safety and Health, Center for Disease Control and Prevention, Cincinnati, 98, 145-149.

Dan, H., Falck, A., \& Ortengren, R. (2010). The Impact of Poor Assembly Ergonomics on Product Quality : A Cost - Benefit Analysis in Car Manufacturing, 20(1), 24-41. http://doi.org/10.1002/hfm

Dance, D., Williams, W., Veltri, A., \& Tx, A. (1996). Application of Cost of Owners to Environment, Safeth and Health, 237-241.

De Greef, M., \& Van den Broek, K. The Case for Workplace Health Promotion (2004).

Hasle, P., Bojesen, A., Jensen, P. L., \& Bramming, P. (2012). Lean and the working environment: a review of the literature.

Kirsten, W. (2010). Making the Link between Health and Productivity at the Workplace-A Global Perspective. Industrial health, 48(3), 251-255

Inail-Contarp. (2012). The data on the OHSMS effectiveness to reduce accidents.

ILO International Labour Office (2010). Ergonomic checkpoints Practical and easy-to-implement solutions for improving safety, health and working conditions.
International Labour Organization 2010 First published 1996; Second edition 2010.

ISSA (International Social Security Association). (2011). The return on prevention: Calculating the costs and benefits of investments in occupational safety and health in companies, (Geneva, Switzerland).

Nieuwenhuis, M., \& Lyons, M. (2002, July 7). Health and Safety Issues and Perceptions of Forest Harvesting Contractors in Ireland. International Journal of Forest Engineering. Retrieved from http://journals.hil.unb.ca/index.php/IJFE/article/view/989 $6 / 10070$

Podgórski, D. (2015). Measuring operational performance of OSH management system - A demonstration of AHPbased selection of leading key performance indicators. Safety Science, 73, 146-166. http://doi.org/10.1016/j.ssci.2014.11.018

Shearn. (2003). Case Examples: Business Benefits Arising From Health \& Safety Interventions.

Tompa, E., Dolinschi, R., de Oliveira, C., \& Irvin, E. (2009). A systematic review of occupational health and safety interventions with economic analyses. Journal of Occupational and Environmental Medicine / American College of Occupational and Environmental Medicine, 51(9), 1004-23. http://doi.org/10.1097/JOM.0b013e3181b34f60

Veltri, A., Pagell, M., Johnston, D., Tompa, E., Robson, L., Amick III, B. C., ... \& Macdonald, S. (2013). Understanding safety in the context of business operations: An exploratory study using case studies. Safety science, 55, 119-134

Von Ahsen, A. (2013). The Integration of Quality, Environmental and Health and Safety Management by Car Manufacturers - a Long-Term Empirical Study. Business Strategy and the Environment, n/a-n/a.

Zohar, D., 2000. A group level model of safety climate: testing the effects of group climate on micro-accidents in manufacturing jobs. Journal of Applied Psychology 85 (4), 587-596.

Zwetsloot, G. I. (1995). Improving cleaner production by integration into the management of quality, environment and working conditions. Journal of Cleaner Production, 3(1), 61-66. 